\title{
Bend loss in surface plasmon polariton band-gap structures
}

\author{
Bozhevolnyi, S.I.; Volkov, V.S.; Leosson, Kristjan; Boltasseva, Alexandra
}

Published in:

Applied Physics Letters

Link to article, DOI:

$10.1063 / 1.1389507$

Publication date:

2001

Document Version

Publisher's PDF, also known as Version of record

Link back to DTU Orbit

Citation (APA):

Bozhevolnyi, S. I., Volkov, V. S., Leosson, K., \& Boltasseva, A. (2001). Bend loss in surface plasmon polariton band-gap structures. Applied Physics Letters, 79(8), 1076-1078. https://doi.org/10.1063/1.1389507

\section{General rights}

Copyright and moral rights for the publications made accessible in the public portal are retained by the authors and/or other copyright owners and it is a condition of accessing publications that users recognise and abide by the legal requirements associated with these rights.

- Users may download and print one copy of any publication from the public portal for the purpose of private study or research.

- You may not further distribute the material or use it for any profit-making activity or commercial gain

- You may freely distribute the URL identifying the publication in the public portal

If you believe that this document breaches copyright please contact us providing details, and we will remove access to the work immediately and investigate your claim. 


\title{
Bend loss in surface plasmon polariton band-gap structures
}

\author{
Sergey I. Bozhevolnyi ${ }^{\mathrm{a})}$ and Valentyn S. Volkov \\ Institute of Physics, Aalborg University, Pontoppidanstrade 103, DK-9220 Aalborg, Denmark \\ Kristjan Leosson and Alexandra Boltasseva \\ Research Center COM, Technical University of Denmark, Building 349, DK-2800 Lyngby, Denmark
}

(Received 2 March 2001; accepted for publication 4 June 2001)

\begin{abstract}
Using near-field optical microscopy, we investigate propagation of surface plasmon polaritons (SPPs) excited in the wavelength range of $720-830 \mathrm{~nm}$ at a corrugated gold-film surface with areas of 200-nm-wide and 45-nm-high scatterers arranged in a 410-nm-period triangular lattice containing line defects with double bends. We find that, for $\sim 2-\mu \mathrm{m}$-wide line defects and the wavelength of $\sim 740 \mathrm{~nm}$, the double bend losses for bend angles of $15^{\circ}$ and $30^{\circ}$ are below 2 and $10 \mathrm{~dB}$, respectively. Our data indicate that the bend loss increases approximately quadratically with the bend angle. We also demonstrate splitting and combining of two SPP line-defect modes in a 20- $\mu$ m-long $Y$ junction. (C) 2001 American Institute of Physics. [DOI: 10.1063/1.1389507]
\end{abstract}

Conventional photonic band-gap (PBG) structures, also called photonic crystals, consist of regions having a periodically modulated (in space) refractive index so that the propagation of light is inhibited in a certain interval of wavelengths. ${ }^{1}$ The great interest in PBG structures is largely driven by the possibility of efficient (even lossless) waveguiding around a sharp corner of a line defect in the PBG structure. ${ }^{2}$ The obvious implication of this feature is an alternative approach to realization of photonic circuits with an unprecedented level of integration. ${ }^{3}$ Examples of successfully realized two-dimensional (2D), PBG-based, optical components include a defect-mode laser, ${ }^{4}$ microcavities, ${ }^{5}$ highly dispersive elements ("superprisms"), ${ }^{6}$ and waveguide bends. ${ }^{7}$ Inspired by the demonstration ${ }^{8}$ of the (complete) band-gap effect for surface plasmon polaritons (SPPs) and our own activity ${ }^{9}$ within micro-optics of SPPs, we have recently suggested to employ SP band-gap (SPPBG) structures for photonic circuits and demonstrated the SPP guiding along line defects in SPPBG structures. ${ }^{10}$

In this letter, we report the observations of guiding around corners of SPP line-defect modes in SPPBG structures. SPPs are quasi-2D (surface) electromagnetic waves propagating along a metal-dielectric interface and having amplitudes exponentially decaying in the neighbor media. ${ }^{11}$ Any surface defect scatters the incident SPP wave into other (diverging) SPPs, which is a $2 \mathrm{D}$ scattering process opening up possibilities for SPP manipulation. ${ }^{9}$ It also scatters the incident SPP into propagating away from the surface field components, which is unwanted scattering giving rise to SPP loss. Unfortunately, even the SPP scattering by an individual surface defect is a rather complicated phenomenon, ${ }^{12}$ and very little is known about SPPBG structures on the theoretical side. Note that, contrary to what might have been expected, ${ }^{2}$ the SPP guiding observed so far did not show the effect of turning around sharp corners. ${ }^{10}$ It is, therefore, of utmost importance to determine the level of bend loss in the SPPBG structures.

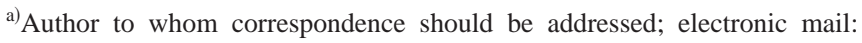
sergey@physics.auc.dk
}

The sample used in this work has been prepared by evaporating a 45-nm-thick gold film on a glass substrate and covering the film surface with areas of a 410-nm-period triangular lattice of 200-nm-wide and 45-nm-high gold scatterers. ${ }^{10}$ The fabricated surface structure contained $\sim 25$ $\mu \mathrm{m}$-long and $\sim 2-\mu \mathrm{m}$-wide line defects with two main orientations, viz., $\Gamma K$ and $\Gamma M$, of the irreducible Brillouin zone of the lattice. ${ }^{1}$ These line defects featured different double bends (the distance between the bends is $\sim 4.5 \mu \mathrm{m}$ ) with angles varying from $0^{\circ}$ to $30^{\circ}$ in steps of $5^{\circ}$. The experimental setup (Fig. 1) is similar to that used in the previous experiments, ${ }^{9}$ and consists of a scanning near-field optical microscope ${ }^{13}$ and an arrangement for SPP excitation in the Kretschmann configuration. ${ }^{11}$ The $p$-polarized (the electric field is parallel to the plane of incidence) light beam from a Ti:sapphire laser $(\lambda=720-830 \mathrm{~nm}, P \sim 100 \mathrm{~mW})$ is weakly focused (spot size $\sim 300 \mu \mathrm{m}$ ) onto the sample attached with immersion oil to the base of a glass prism. The SPP excitation is recognized as a minimum in the angular dependence of the reflected light power. For a given angle, the SPP excitation remains close to optimum within $\sim 30 \mathrm{~nm}$ of wavelength variation, but outside of this interval the angle of the

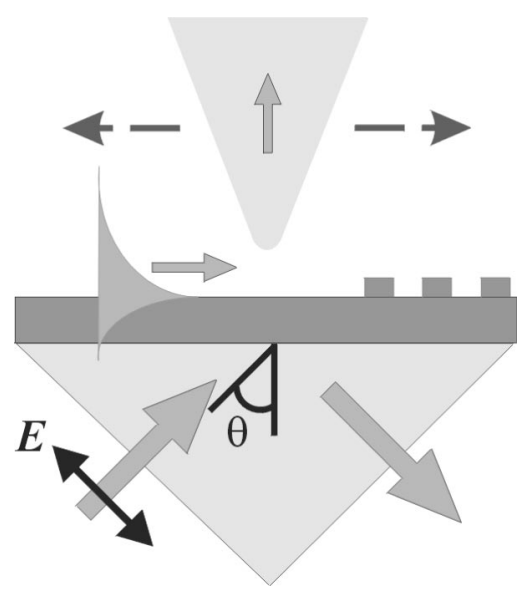

FIG. 1. Schematic representation of the excitation of surface plasmon polaritons and their detection with an uncoated fiber tip of a collection nearfield microscope. Polarization of the incident laser beam is also indicated. 


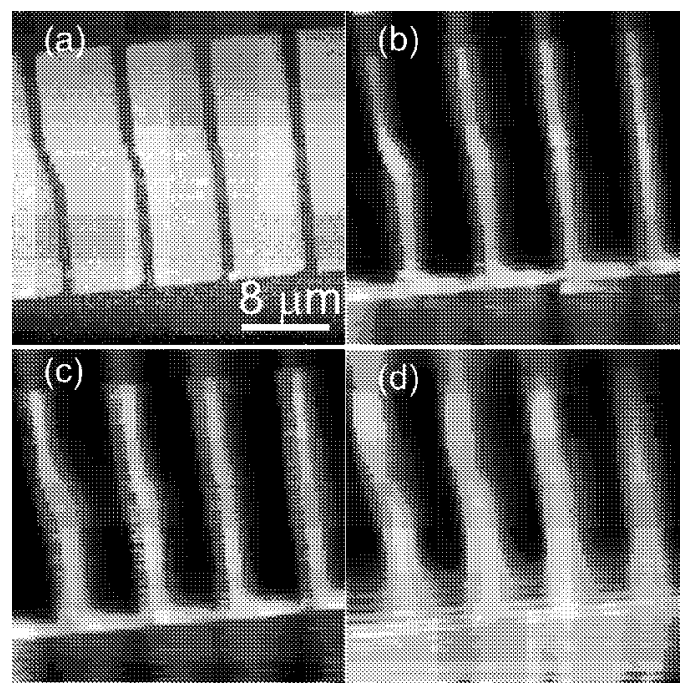

FIG. 2. Gray-scale (a) topographical and near-field optical images (32 $\times 32 \mu \mathrm{m}^{2}$ ) taken at $\lambda \cong$ (b) 737 , (c) 726 , and (d) $795 \mathrm{~nm}$. Periodic surface structure is of $\Gamma M$ orientation and contains a straight line defect followed (from right to left) by line defects with $5^{\circ}, 10^{\circ}$, and $15^{\circ}$ double bends.

laser beam incidence has to be readjusted. The SPP propagation length evaluated at the wavelength of $800 \mathrm{~nm}$ for an air-gold interface is $\sim 35 \mu \mathrm{m}$. ${ }^{11}$ In the configuration used, it gets further reduced due to the radiative damping (resonant reradiation in the substrate) and inelastic SPP scattering out of the surface plane. The SPP excitation exhibited a wellpronounced resonance behavior with respect to the variations in the angle of light incidence. The average optical signal also decreased drastically when changing the angle of incidence and with the increase of the tip-surface distance. This means that the optical images recorded with the tip-surface distance being maintained by shear force feedback reflect primarily the total SPP field intensity distribution along the film surface. ${ }^{9}$ Finally, it should be noted that the images presented here (Figs. 2, 3, and 5) are oriented in a way that the excited SPP propagates upwards in the vertical direction.

The efficient SPP guiding along the straight $\sim 2-\mu \mathrm{m}-$ wide and $24-\mu \mathrm{m}$-long line defect (with $\Gamma M$ orientation) in the SPPBG structure [Fig. 2(a)] has been observed in the wavelength range of $730-750 \mathrm{~nm}$ with the best near-field optical image being obtained at $\lambda \cong 737 \mathrm{~nm}$ [Fig. 2(b)]. It is seen that the SPP intensity is strongly damped inside the SPPBG structure, whereas the defect mode maintains its amplitude during the propagation in the structure. The first feature is explained by the fact that the SPP propagation inside the periodically corrugated surface region is inhibited for light wavelengths corresponding to the SPPBG. ${ }^{10}$ The second feature indicates that the propagation constant of the defect mode is close to that of the SPP and, therefore, the mode power loss due to the damping and scattering can be compensated by the coupling from the incident laser beam (adjusted to excite the SPP outside the SPPBG structure). The confinement of the defect mode and SPP damping inside the periodic structure gradually deteriorated with the decrease [Fig. 2(c)] and increase of the light wavelength [Fig. 2(d)], thus providing clear and direct evidence of the SPPBG effect in the structure.

The SPP guiding through double bends (at the wavelength of $737 \mathrm{~nm}$ ensuring the best mode confinement) was Downloaded 24 Feb 2010 to 192.38.67.112. Redistribution subject
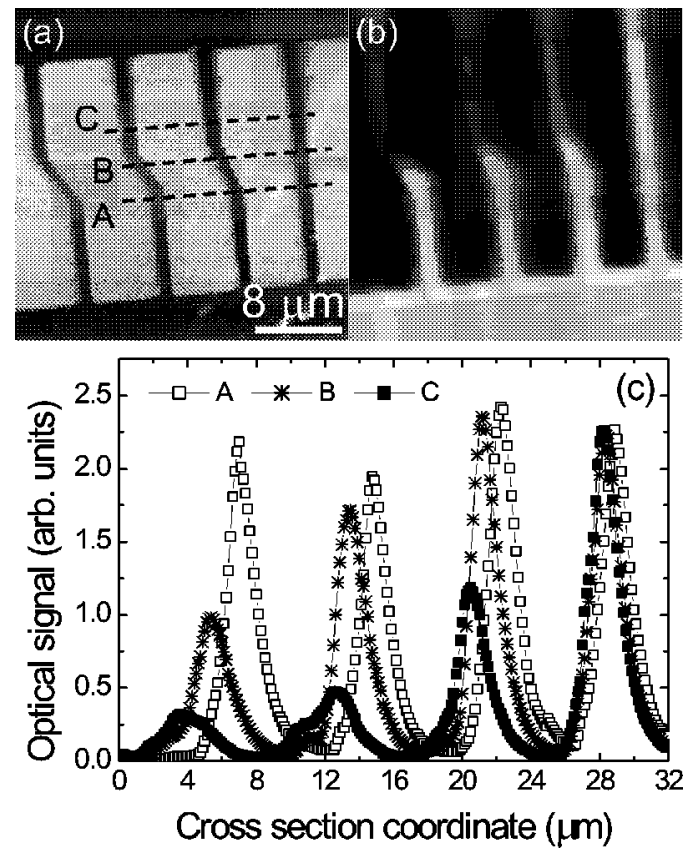

FIG. 3. Gray-scale (a) topographical and (b) near-field optical images (32 $\times 32 \mu \mathrm{m}^{2}$ ) taken at $\lambda \cong 737 \mathrm{~nm}$. Periodic surface structure is of $\Gamma M$ orientation and contains a straight line defect followed (from right to left) by line defects with $20^{\circ}, 25^{\circ}$, and $30^{\circ}$ double bends. (c) Cross sections of the optical image shown in Fig. 2(b) at different positions with respect to the double bends as shown in Fig. 2(a).

found to be markedly deteriorating with the increase of the bend angle from $5^{\circ}$ to $30^{\circ}$ [Figs. 2(b) and 3(b)]. Note that the straight line defect in the second structure exhibits the same unhindered mode propagation as that in the first one, indicating that the excitation conditions are similar in both cases and thereby justifying the comparison of the SPP guiding around bends in these structures. The cross sections of the corresponding near-field optical images [e.g., Fig. 3(c)] showed that the mode profile is preserved during and after guiding around bends (with the intensity mode half width at the $1 / e^{2}$ level being $\sim 2 \mu \mathrm{m}$ ). This circumstance allows one to determine the bend loss by simply relating the maximums of the intensity mode profiles, which are obtained by averaging several cross sections made at the input and at the output of a double bend.

The results of the aforementioned procedure carried out for the investigated SPPBG structures with $\Gamma M$ [Figs. 2(b) and $3(\mathrm{~b})]$ and $\Gamma K$ (not shown) orientations are plotted in Fig. 4 together with a quadratic fit of all data obtained. The latter was impelled by the circumstance that, in the limit of small angles, fundamental modes of conventional channel waveguides and fibers experience the bend loss proportional to the square of the bend angle. ${ }^{14}$ The fit seems reasonably good, but if one makes use of the corresponding formula for Gaussian-shaped modes, ${ }^{14}$ one ends up with the mode half width of $\sim 0.45 \mu \mathrm{m}$, a value which is at least four times smaller than the measured one. Such a discrepancy should evidently be imputed to the fundamental difference between the guiding mechanisms in traditional waveguides and PBG line defects. The phenomenon of total internal reflection, which is responsible for the traditional waveguiding, occurs in a limited range of incident angles depending on the refractive index contrast, whereas the photonic crystals (PBG olP license or copyright; see http://apl.aip.org/apl/copyright.jsp 


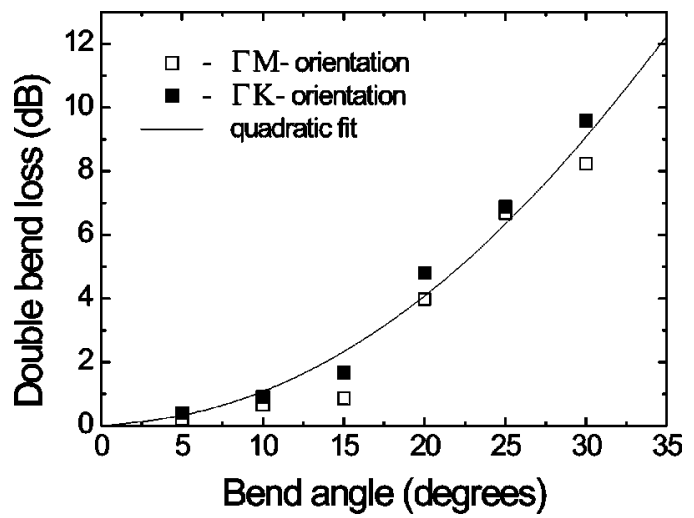

FIG. 4. Double bend loss as a function of the bend angle determined from cross sections of near-field optical images [for example, see Fig. 2(c)] obtained for structure of $\Gamma M$ and $\Gamma K$ orientations. A quadratic fit to all data is shown by a solid line.

structures) would reflect the radiation incident at any angle provided that its wavelength is inside the (complete) PBG. The observed bend loss in the SPPBG structures is probably related to the same circumstance as that in the conventional 2D PBG structures, ${ }^{7}$ viz., that these structures are only quasi$2 \mathrm{D}$, implying the existence of loss by scattering out of the surface plane.

We would like to point out that the loss level attained already in these experiments indicates that the waveguide circuits in the SPPBG structures having relatively large (tens of degrees) bend angles can be realized with relatively small $(<10 \mathrm{~dB})$ losses, allowing for a high integration level. As a first example, we have fabricated and investigated a $20^{\circ} \mathrm{Y}$ junction in the SPPBG structure of $\Gamma M$ orientation with the same parameters as those described above [Fig. 5(a)]. It is seen that splitting/combining of two SPPBG line-defect modes is accomplished over the length of $\sim 20 \mu \mathrm{m}$ [Fig. 5(b)]. For the $Y$ splitter, the power loss does not exceed $3 \mathrm{~dB}$ [Fig. 5(c)] and at least some of the loss should be attributed to the propagation loss due to (internal and radiative) damping and scattering (out of the surface plane).

In summary, we have investigated the bend loss for SPP modes (at the wavelength of $\sim 740 \mathrm{~nm}$ ) propagating along $\sim 2$ - $\mu$ m-wide line defects in 410-nm-period SPPBG structures fabricated at the surface of 45-nm-thick gold film. We have found that the double bend loss is below $2 \mathrm{~dB}$ for the bend angle of $15^{\circ}$ and increases approximately quadratically with the bend angle. Finally, we have demonstrated the possibility of splitting and combining of two SPPBG line-defect modes over the distance of $\sim 20 \mu \mathrm{m}$, thereby rendering the prospect of high integration of the SPPBG-based photonic circuits viable. We believe that further investigations and optimization of the structural parameters will allow us to decrease the level of bend loss in the SPPBG structures, and we continue our work in this direction.
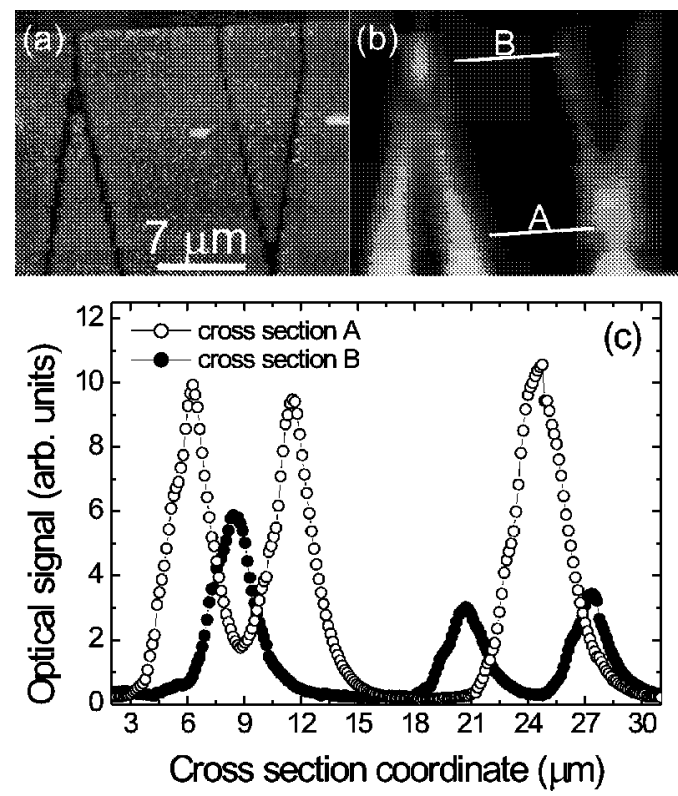

FIG. 5. Gray-scale (a) topographical and (b) near-field optical images (28 $\times 22.5 \mu \mathrm{m}^{2}$ ) taken at $\lambda \cong 737 \mathrm{~nm}$. Periodic surface structure is of $\Gamma M$ orientation and contains two facing each other $20^{\circ} Y$ junctions. (c) Cross sections of the optical image shown in Fig. 4(b) at different positions with respect to the $Y$ junctions.

The authors gratefully acknowledge financial support from the Danish Technical Research Council under Contract No. 56-00-0329. Electron-beam lithography has been performed in the III-V Nanolab, a joint laboratory between Research Center COM and the Niels Bohr Institute, Copenhagen University.

${ }^{1}$ J. D. Joannopoulos, R. D. Meade, and J. N. Winn, Photonic Crystals (Princeton University Press, Princeton, NJ, 1995).

${ }^{2}$ A. Mekis, J. C. Chen, I. Kurland, S. Fan, P. R. Villeneuve, and J. D. Joannopoulos, Phys. Rev. Lett. 77, 3787 (1996).

${ }^{3}$ T. F. Krauss and R. M. De La Rue, Prog. Quantum Electron. 23, 51 (1999).

${ }^{4}$ O. Painter, R. K. Lee, A. Scherer, A. Yariv, J. D. O’Brien, P. D. Dapkus, and I. Kim, Science 284, 1819 (1999).

${ }^{5}$ C. J. M. Smith, H. Benisty, D. Labilloy, U. Oesterle, R. Houdré, T. F. Krauss, R. M. De La Rue, and C. Weisbuch, Electron. Lett. 35, 228 (1999).

${ }^{6}$ H. Kosaka, T. Kawashima, A. Tomita, M. Notomi, T. Tamamura, T. Sato, and S. Kawakami, Appl. Phys. Lett. 74, 1370 (1999).

${ }^{7}$ M. Tokushima, H. Kosaka, A. Tomita, and H. Yamada, Appl. Phys. Lett. 76, 952 (2000); M. Loncar, D. Nedeljkovic, J. Vuckovic, A. Scherer, and T. P. Pearsall, ibid. 77, 1937 (2000).

${ }^{8}$ S. C. Kitson, W. L. Barnes, and J. R. Sambles, Phys. Rev. Lett. 77, 2670 (1997)

${ }^{9}$ S. I. Bozhevolnyi and F. A. Pudonin, Phys. Rev. Lett. 78, 2823 (1997); S. I. Bozhevolnyi and V. Coello, Phys. Rev. B 58, 10899 (1998).

${ }^{10}$ S. I. Bozhevolnyi, J. Erland, K. Leosson, P. M. W. Skovgaard, and J. M. Hvam, Phys. Rev. Lett. 86, 3008 (2001).

${ }^{11}$ H. Raether, Surface Plasmons (Springer, Berlin, 1988).

${ }^{12}$ A. V. Shchegrov, I. V. Novikov, and A. A. Maradudin, Phys. Rev. Lett. 78, 4269 (1997).

${ }^{13}$ DME-DualScope ${ }^{\mathrm{TM}}$, Herlev, Denmark.

${ }^{14} \mathrm{~A}$. Ghatak and K. Thyagarajan, Introduction to Fiber Optics (Cambridge University Press, Cambridge, U.K., 1998). 